\title{
Testing Pauli Exclusion Principle for electrons at the LNGS underground laboratory: the VIP-2 experiment
}

Luca De Paolis ${ }^{a, *}$, Fabrizio Napolitano $^{a}$, Kristian Piscicchia $^{b}$, Sergio Bartalucci $^{a}$, Sergio Bertolucci ${ }^{e}$, Massimiliano Bazzi $^{a}$, Mario Bragadireanu ${ }^{a, d}$, Michael Cargnelli ${ }^{c}$, Alberto Clozza $^{a}$, Raffaele Del Grande ${ }^{f, a}$, Carlo Fiorini ${ }^{g}$, Carlo Guaraldo ${ }^{a}$, Mihai Iliescu $^{a}$, Matthias Laubenstein ${ }^{h}$, Johann Marton $^{c}$, Marco Miliucci $^{a}$, Edoardo Milotti ${ }^{i}$, Alessio Porcelli ${ }^{c, a}$, Alessandro Scordo $^{a}$, Francesco Sgaramella $^{a}$, Hexi Shi ${ }^{c}$, Diana Laura Sirghi $^{a, d}$, Florin Sirghi ${ }^{a, d}$, Johann Zmeskal ${ }^{c}$, Catalina Curceanu ${ }^{a}$

a INFN, Laboratori Nazionali di Frascati, Via E. Fermi 54, I-00044 Frascati(RM), Italy;

${ }^{b}$ Centro Ricerche Enrico Fermi-Museo Storico della Fisica e Centro Studi e Ricerche "Enrico Fermi”,

Via Panisperna 89A 00184, Rome, Italy;

${ }^{c}$ Stefan Meyer Institute for Subatomic Physics, Kegelgasse 27, 1030 Wien, Austria;

${ }^{d}$ Horia Hulubei National Institute of Physics and Nuclear Engineering, Str. Atomistilor No. 407, P.O. Box MG-6 Buchares-Magurele, Romania;

e Dipartimento di Fisica e Astronomia, University of Bologna and INFN-Sezione di Bologna, Via Irnerio 46, I-40126 Bologna, Italy;

${ }^{f}$ Excellence Cluster Universe, Technische Universit ä M ̈unchen, Boltzmannstraße 2, 85748 Garching bei M̈̈unchen, Germany;

g Politecnico di Milano, Dipartimento di Elettronica, Informazione e Bioingegneria and INFN Sezione di Milano, I-20133 Milano, Italy;

${ }^{h}$ INFN, Laboratori Nazionali del Gran Sasso, Via G. Acitelli 22, I-67100 L'Aquila, Italy;

${ }^{i}$ Dipartimento di Fisica, Università di Trieste and INFN-Sezione di Trieste, Via Valerio, 2, I-34127

Trieste, Italy;

E-mail: Luca.DePaolis@lnf.infn.it, Napolitano.Fabrizio@lnf.infn.it

\footnotetext{
*Speaker
} 
The VIP-2 experiment tests the Pauli Exclusion Principle (PEP) for electrons at the Gran Sasso underground National Laboratories (LNGS) of INFN in Italy, looking for a possible violation. The LNGS provide an extremely low background environment, ideal for performing high precision $\mathrm{X}$-ray spectroscopy measurements on electrons atomic transitions. The core of the VIP-2 experimental apparatus is based on a copper target circulated by a Direct Current (DC) and surrounded by silicon drift detectors (SDDs), which offer excellent performance in X-ray spectroscopy in the energy range experimentally observed by VIP-2. The aim of VIP-2 is to look for possible PEPforbidden $\mathrm{K} \alpha$ transitions ( $2 \mathrm{p} \rightarrow 1 \mathrm{~s}$ ) in copper atoms, when the $1 \mathrm{~s}$ level would be already occupied by two electrons, in contradiction with PEP. The energy of the $\mathrm{K} \alpha$ forbidden transitions is about $300 \mathrm{eV}$ less than the nominal energy of the K $\alpha$ PEP-allowed transition. This energy shift is due to the screening effect produced by the extra electron in the fundamental level, and is detectable through a high precision X-ray spectroscopy measurement. The precedent VIP experiment set the best upper limit on the PEP violation probability $\beta^{2} / 2<4.7 \times 10^{-29}$ for electrons. The goal of the VIP-2 experiment is to improve this limit by two orders of magnitude. This paper presents a new preliminary result, obtained by analysing two sets of data collected with a partial configuration of the VIP-2 apparatus. 


\section{Introduction}

The Pauli Exclusion Principle (PEP), which states that two identical fermions cannot simultaneously occupy the same quantum state [1], represents a fundamental pillar of quantum mechanics. Particles can be grouped into bosons having integer spin and fermions having half-integer spin. Bosonic states are symmetrical concerning the application of the permutation transformation (i.e., exchange of identical particles), and, conversely, fermionic states are antisymmetrical [2]. The Messiah-Greenberg (MG) superselection rule [3] forbids transitions between different symmetry states. Therefore, any experimental evidence of PEP violation would result in particles following a different statistic than the fermionic and bosonic ones and could lead to a new physics beyond the Standard Model. The VIP-2 experiment searches for K $\alpha$ PEP-violating transition ( $2 p \rightarrow 1 s)$ in copper with high precision X-rays detectors. This atomic transition is expected to be shifted down by $300 \mathrm{eV}$ due to the screening effect produced by the additional electron already occupying the $1 \mathrm{~s}$ atomic level [6], i.e., at $7746.73 \mathrm{eV}$. The goal of the VIP-2 experiment is to improve the upper limit on the Pauli Exclusion Principle violation probability of $\beta^{2} / 2<4.7 \times 10^{-29}$ [4], previously set by the VIP experiment, by two orders of magnitude.

\section{The VIP-2 experimental apparatus}

The VIP-2 new apparatus is presently taking data at Gran Sasso underground National Laboratory(LNGS), which provides an extremely low cosmic background environment. The target consists of 2 strips of copper $(71 \mathrm{~mm} \times 20 \mathrm{~mm} \times 25 \mu \mathrm{m})$. A Direct Current (DC) of $100 \mathrm{~A}$ is circulated on the target strips. For X-ray detection, 32 Silicon Drift Detector (SDD) cells were installed around the target. The SDDs are cooled to $150 \mathrm{~K}$ with liquid argon and, in these working conditions, provide an energy resolution is $190 \mathrm{eV} \mathrm{FWHM}$ at $\sim 8 \mathrm{keV}$ and an X-ray detection efficiency of about $99 \%$. Each SDD cell has $0.64 \mathrm{~cm}^{2}$ of active area and a timing resolution of $400 \mathrm{~ns}$. The elements of the apparatus described above are placed inside a vacuum chamber. The pressure is kept fixed at $10^{-5}$ mbar because of the SDD cooling system [7]. In addition, an external shielding was installed for further background reduction, consisting of an internal layer of copper bricks and an exterior layer of lead bricks outside the apparatus. More details on the VIP-2 apparatus can be found in [5].

\section{The data analysis: procedure and new preliminary result}

In the VIP-2 experiment, part of the data is collected with a DC circulating on target, thus introducing newly-injected electrons which interact with copper atoms in an Open System that fulfills the Messiah-Greenberg superselection rule [8]. In addition, spectra with no current circulating on the target are acquired as background references.

In this paper, we analyze two sets of data in a non-final configuration of the VIP-2 apparatus. The first set consists of 42 days of data taking with 100 A circulating on target and 65 days without current circulating on target, collected before the external shielding installation on the setup. The second set consists of 40 days of data taking with 100 A circulating on target and 61 days without current circulating on target, collected with only lateral part of the external shielding installed around the setup. The spectra are shown in Fig. 1.

A two-facet approach was employed to analyze the data. Since the data taking is operated in two different experimental conditions and detectors, we perform both a Bayesian and frequentist analysis based on a binned likelihood function, considering the two separate datasets, using RooFit and 

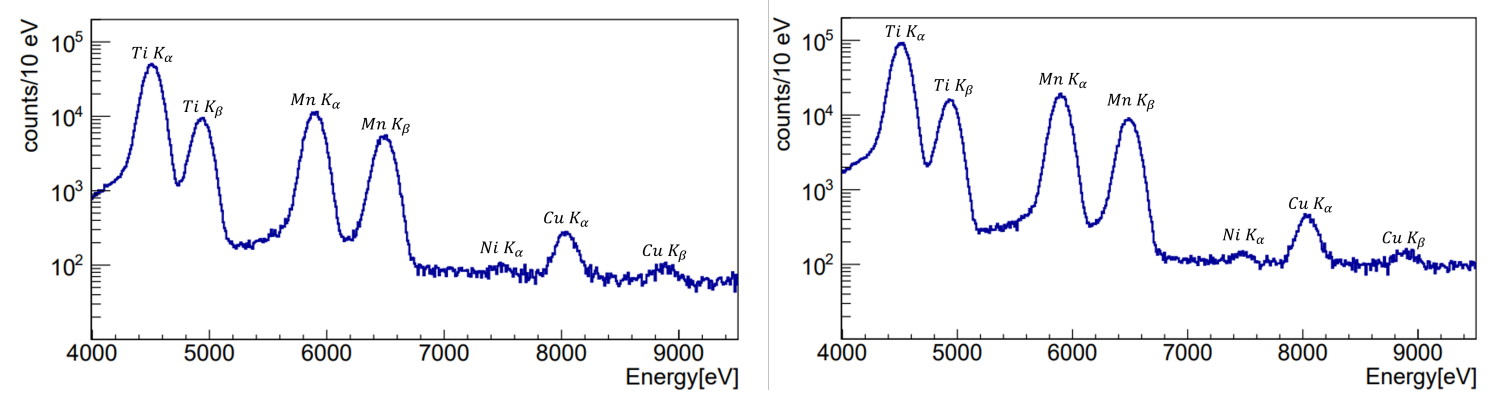

Figure 1: Spectra collected with partial shielding configuration (only lateral part). On the left, with $100 \mathrm{~A}$ circulating on target; On the right, without current. In the spectra, titanium and manganese lines come from the Fe-55 radioactive source installed in the apparatus for calibration; the nickel line comes from the detectors' ceramic support.

RooStat [10]. For this preliminary result, we use the formalism of a counting "on/off" experiment. The counts are selected in the Region Of Interest (ROI): 7647-7847 eV. The background in the data with current ("on") is constrained by the data without current ("off") in each dataset. The number of the signal events in the data acquired with current can be written as $S_{i}=F_{i} \times \epsilon_{i} \times \beta^{2} / 2$, where the $i$ index runs over the two datasets $D_{i}$ for $i=1,2$. The $F_{i}$ depends only on the data acquisition time with current $t_{i}^{w c}$ and a common factor depending on the current, mean free path of the electrons in copper, absorption probability, and target dimensions. Finally, $\epsilon_{i}$ depends on the number of SDDs used in each $D_{i}$ and their Monte Carlo efficiency. The likelihood function can be simply written as:

$$
\mathcal{L}\left(D_{i} \mid S_{i}, B_{i}, S C A L E_{i}\right)=\operatorname{Poiss}\left(N_{i}^{w c} \mid S_{i}+B_{i}\right) \times \operatorname{Poiss}\left(N_{i}^{w o c} \mid B_{i} \times S C A L E_{i}\right),
$$

where Poiss $(n \mid x)=\frac{x^{n}}{n !} e^{-x}, N_{i}^{w c}$ and $N_{i}^{w o c}$ are the number of events in the $D_{i}$ for the spectrum with current and without current respectively, $B_{i}$ expected background in $D_{i}$ and finally $S C A L E_{i}$ a factor which depends on the ratio $t_{i}^{w c} / t_{i}^{w o c}$ used to rescale $B_{i}$ from the data without current to the data with the current. The systematic uncertainty on $S C A L E_{i}$ is folded into the likelihood by adding multiplicative Gaussian penalty terms. A frequentist analysis is performed using a one-sided test statistics [9], and we used $C L_{s}=\frac{C L_{s+b}}{1-C L_{b}}$ at $90 \%$ confidence level. A Bayesian result using the same likelihood is also at $90 \%$ confidence using a flat prior for $\beta^{2} / 2$. Using Monte Carlo Markov Chain numerical integration, all the parameters are marginalized to obtain the one-dimensional posterior PDF of $\beta^{2} / 2$. The observed upper limit on $\beta^{2} / 2$ is $4.3^{-30}$ for both the frequentist CLs and Bayesian treatment.

\section{Conclusions}

Two sets of VIP-2 data of 107 and 101 days, respectively with no and partial shielding, were used to obtain preliminary upper limits on the $\beta^{2} / 2$ probability of violation of the PEP. The data was analyzed with a frequentist and a Bayesian analysis yielding $\beta^{2} / 2<4.3 \times 10^{-30}$ at $90 \%$ confidence level. That is the stronger limit to date set by the VIP-2 experiment, using 2019 data with incomplete setup. After this measurement, the external shielding was completed, and the VIP-2 experiment started the data taking campaign in its final configuration. The data taking is presently ongoing with the aim to further improve our limit on the PEP violation probability. To this end, advanced data analysis techniques are under development. 


\section{Acknowledgments}

This publication was made possible through the support of Grant 62099 from the John Templeton Foundation. The opinions expressed in this publication are those of the authors and do not necessarily reflect the views of the John Templeton Foundation. We acknowledge support from the Foundational Questions Institute and Fetzer Franklin Fund, a donor advised fund of Silicon Valley Community Foundation (Grants No. FQXi-RFP-CPW-2008 and FQXi-MGB-2011), and from the H2020 FET TEQ (Grant No. 766900) and INFN (VIP). We thank the Austrian Science Foundation (FWF) which supports the VIP-2 project with the grants P25529-N20, project P 30635-N36 and W1252-N27 (doctoral college particles and interactions).

\section{References}

[1] I. G. Kaplan, The Pauli Exclusion Principle and the Problems of Its Experimental Verification., Symmetry 12 (320) 2020

[2] R. Hilborn and G. Tino, Spin-Statistics Connection and Commutation Relations: Experimental Tests and Theoretical Implications., AIP Conference Proceedings American Institute of Physics: Melville, NY, USA 2000

[3] A. Messiah and O. Greenberg, Symmetrization Postulate and Its Experimental Foundation, Physical Review 136 (B248) 1964

[4] C. Curceanu et al., Experimental tests of quantum mechanics - Pauli exclusion principle violation (the VIP experiment) and future perspective, J. Phys. Conf. Ser. 306 (012036) 2011

[5] L. De Paolis et al., The key role e of the Silicon Drift Detectors in testing the Pauli Exclusion Principle for electrons: the VIP2 experiment J. Phys.: Conf. Ser. 1548 (012033) 2020

[6] C. Curceanu et al., EVALUATION OF THE X-RAY TRANSITION ENERGIES FOR THE PAULI-PRINCIPLE-VIOLATING ATOMIC TRANSITIONS IN SEVERAL ELEMENTS BY USING THE DIRAC-FOCK METHOD, INFN Technical Note Report INFN-13-21 (LNF) 2013

[7] H. Shi et al., Experimental search for the violation of Pauli exclusion principle, Eur. Phys. J. C 78 (319) 2018

[8] K. Piscicchia et al., High precision test of the Pauli Exclusion Principle for electrons, Eur. Phys. J. C 1586 (012016) 2020

[9] Cowan, G. et al. Asymptotic formulae for likelihood-based tests of new physics. Eur. Phys. J. C 71, 1554 (2011).

[10] Rene Brun and Fons Rademakers, ROOT - An Object Oriented Data Analysis Framework, Proceedings AIHENP'96 Workshop, Lausanne, Sep. 1996, Nucl. Inst. \& Meth. in Phys. Res. A 389 (1997) 81-86. 